\title{
Special issue: ENSO diversity
}

\section{Benjamin Kirtman ${ }^{1}$}

Published online: 2 April 2019

(c) Springer-Verlag GmbH Germany, part of Springer Nature 2019

It has long been known that each ENSO event is unique. However, it is only in the last 10-15 years that the importance of these event-to-event differences for atmospheric teleconnections and societally-relevant impacts has been acknowledged, promoting a more systematic classification of the different types of El Niño events. Research efforts have primarily focused in separating ENSO events into two groups: the "canonical" El Niño events, which are characterized by warmest sea surface temperature anomalies (SSTAs) in the Eastern Pacific (EP), and the so-called Central Pacific (CP) El Niño events, which have their largest SSTA near the Dateline. Several indices and definitions have been introduced to describe these two classes of events, and more easily identify their leading dynamical feedbacks and remote impacts. For example, the growth and decay of EP events in the eastern equatorial Pacific is controlled by the so called "thermocline feedback", involving the vertical advection of anomalous vertical temperature gradients by the mean upwelling, while the evolution of CP events is dominated by the "zonal advective" feedback, namely the anomalous zonal advection of the mean temperature gradient. These differences in the leading dynamical processes have led some researchers to view these two classes of events as distinct dynamical entities, while other studies describe ENSO diversity as a continuum of expressions of the complex and multi-faceted ENSO phenomenon.

Apart from the debate on the nature of ENSO diversity, whether bimodal or continuum, many key questions remain unanswered. While the differences in the spatial patterns of ENSO events are expected to result in different atmospheric teleconnections, the exact expression of these differences, and how they translate into remote impacts is not well understood. The complexity of this problem partly originates from the high sensitivity of the ENSO-related rainfall anomalies on the background state, so that weaker SSTAs associated with $\mathrm{CP}$ events may have a large impact on rainfall and teleconnections due to the warmer central/western Pacific background state. What determines ENSO diversity? Are there specific "precursors" or triggers for different event types, or are these differences happening by chance? And how would the existence of these precursors impact our ability to predict these different events? Our current seasonal to interannual prediction systems perform better in predicting EP events, but it is not clear whether the difficulty in predicting $\mathrm{CP}$ events is associated with model deficiencies or with limitations in these event's inherent predictability. Do we have the right observations and observing systems necessary to address the predictability and prediction issues? The physical mechanisms for El Niño and La Niña events (e.g., thermocline vs zonal advective feedback) may themselves be sensitive to the details of an evolving background state, an aspect that raises the question of how ENSO diversity will respond to decadal and longer term climate variations. These issues are probed in this special collection of research on ENSO diversity.
Benjamin Kirtman

bkirtman@rsmas.miami.edu

1 Rosenstiel School for Marine and Atmospheric Science (RSMAS), Miami, USA 